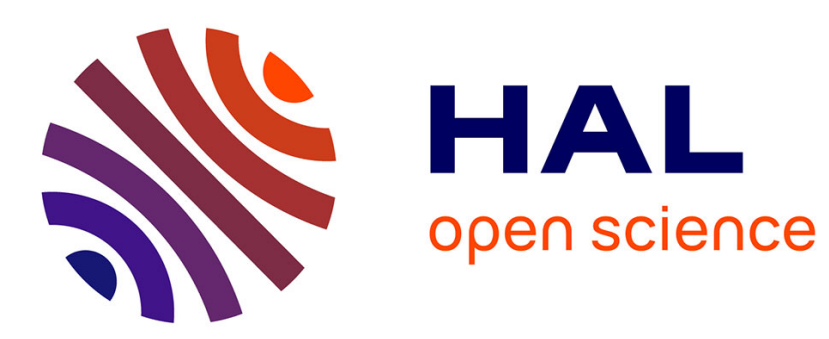

\title{
Cascaded Raman slow light and optical spatial solitons in Kerr media
}

\author{
Gil Fanjoux, Jérémy Michaud, Hervé Maillotte, Thibaut Sylvestre
}

\section{To cite this version:}

Gil Fanjoux, Jérémy Michaud, Hervé Maillotte, Thibaut Sylvestre. Cascaded Raman slow light and optical spatial solitons in Kerr media. Physical Review A: Atomic, molecular, and optical physics [1990-2015], 2013, 87, pp.033838. 10.1103/PhysRevA.87.033838 . hal-00831571

\section{HAL Id: hal-00831571 https://hal.science/hal-00831571}

Submitted on 7 Jun 2013

HAL is a multi-disciplinary open access archive for the deposit and dissemination of scientific research documents, whether they are published or not. The documents may come from teaching and research institutions in France or abroad, or from public or private research centers.
L'archive ouverte pluridisciplinaire HAL, est destinée au dépôt et à la diffusion de documents scientifiques de niveau recherche, publiés ou non, émanant des établissements d'enseignement et de recherche français ou étrangers, des laboratoires publics ou privés. 


\title{
Cascaded Raman slow light and optical spatial solitons in Kerr media
}

\author{
Gil Fanjoux, Jérémy Michaud, Hervé Maillotte, and Thibaut Sylvestre \\ Institut FEMTO-ST, Département d'Optique P.M Duffieux, \\ UMR CNRS-Université de Franche-Comté 6174, Route de Gray, 25030 Besançon cedex, France
}

\begin{abstract}
We provide a complete experimental and numerical investigation of nonlinear beam propagation in a carbon disulfide liquid-filled Kerr slab waveguide using picosecond pulsed light. A first spatial analysis shows the generation of a multicolor solitary wave through the combined effects of cascaded Raman scattering and cross-phase modulation. The impact of temporal effects on this self-trapped optical beam is further studied through a spatiotemporal analysis. It is shown in particular how cascaded Raman slow light induces optical delays between the solitonic components which prevents higher-order spatial soliton and provides a remarkable beam stability. Experimental observations are in very good agreement with numerical simulations of the spatiotemporal (1+1D) nonlinear Schrödinger equation including the Raman scattering.
\end{abstract}

PACS numbers: 42.65.Tg, 42.65.Dr

\section{INTRODUCTION}

Solitons, nonlinear self-trapped wavepackets, continue to attract much attention in various branches of physics such as optics, plasmas, condensed matter physics, fluid mechanics $[1,2]$. In optics, among the wide variety of solitary waves observed in many nonlinear materials, multicolor solitons (MS) are of particular interest because they exist in the spatial and temporal domains $[3,4]$. Multicolor solitons are formed by the mutual trapping of multiple-frequency waves that interact in the nonlinear medium. The simplest case corresponds to the process of second-harmonic generation in quadratic media, in which a fundamental frequency wave and its second harmonic mutually locked and trapped in robust soliton light states that do not spread, either in space or in time [5-8].

Multicolor solitons in cubic media have comparatively been much less investigated than in quadratic media. In isotropic Kerr media, mutual trapping of multiplefrequency waves can be achieved by the cross-phase modulation (XPM) or the four-wave mixing (FWM) [9-12]. MS have also been reported in the spatial domain using stimulated Raman scattering (SRS) in carbon disulfide $\left(\mathrm{CS}_{2}\right)[13,14]$. More precisely, we demonstrated in 2006 the generation of a two-color spatial optical soliton using SRS in a $\mathrm{CS}_{2}$ nonlinear planar waveguide [14]. We shown in particular that the pump and Raman Stokes beams are mutually trapped through both XPM-induced phase shifts and the Raman gain, and that the whole beam keeps its characteristic squared hyperbolic-secant shape during propagation in spite of the energy exchanges due to SRS. A few years later, we reported in the same waveguide the observation of a slow-light spatial soliton, a spatial soliton which has been slowed down by SRS because of its narrow linewidth in $\mathrm{CS}_{2}[15]$. Despite these observations, however, our numerical simulations of the 1D spatial nonlinear Schrödinger equation were not fully consistent with our experimental records, particularly in the depleted pump regime. This difference actually comes from the temporal effects because spatial solitons were generated using picosecond pulsed light and not with continuous-wave (CW) light, as assumed in numerical simulations. Thus we require a more accurate analysis to better understand the spatiotemporal dynamics in such Kerr-type waveguide.

In this paper, we address fully the impact of temporal effects and Raman scattering on the generation of Kerr solitons in a $\mathrm{CS}_{2}$ liquid filled highly nonlinear planar waveguide. It is shown in particular how Raman effect induces optical delays via slow light between the soliton components and provides a remarkable beam stability. Cascaded Raman slow light whereby all Raman orders are mutually delayed by slow light is also clearly evidenced through spatiotemporal measurements. Our experimental measurements are also compared to numerical simulations based on the spatiotemporal (1+1D) nonlinear Schrödinger equation including the Raman scattering.

\section{NUMERICAL MODELING}

To numerically study the spatiotemporal dynamics in a nonlinear planar waveguide, we have developed a $(1+1 \mathrm{D})$ numerical model based on the nonlinear Schrödinger equation (NLSE). This equation includes diffraction in the free transverse dimension $x$ of the planar waveguide, linear dispersion, Kerr nonlinearity and the stimulated Raman scattering (SRS) through its delayed time response $R_{R}(t)$ [15-17]. In the time reference frame of the input electric field, we may write this equation as

$$
\begin{aligned}
& \frac{\partial A}{\partial z}-\frac{i}{2 \beta} \frac{\partial^{2} A}{\partial x^{2}}+\frac{i}{2} \beta_{2} \frac{\partial^{2} A}{\partial t^{2}}= \\
& \quad i \gamma|A|^{2} A+i \gamma A \int_{0}^{\infty} R_{R}(t)\left|A\left(z ; x, t-t^{\prime}\right)\right|^{2} d t^{\prime}
\end{aligned}
$$

where $A(z ; x, t)$ is the slowly varying envelope of the whole electric field propagating in the $z$ direction, $\beta$ the wave-vector, $\beta_{2}$ the group-velocity dispersion (GVD) coefficient and $\gamma=2 \pi n_{2} / \lambda$ the nonlinear coefficient with $n_{2}$ the nonlinear Kerr index and $\lambda$ the pump laser 
wavelength $(\lambda=532 \mathrm{~nm})$. The last integral term accounts for the stimulated Raman scattering in $\mathrm{CS}_{2}$ liquid. It includes both the real and imaginary part of the Raman susceptibility. We assumed for $\mathrm{CS}_{2}$ a Lorentzian-shape narrow linewidth Raman gain with a Raman frequency shift of $20 \mathrm{THz}$ and a linewidth of $15 \mathrm{GHz}$, respectively [18]. The GVD parameter is $\beta_{2}=+4.24 \times 10^{-25} \mathrm{~s}^{2} / \mathrm{m}$, and the nonlinear index $n_{2}=+3.5 \times 10^{-18} \mathrm{~m}^{2} / \mathrm{W}$. We also include an input noise level that corresponds to the quantum noise, i.e., one photon per spatiotemporal mode. SRS starts from this white noise and we have neglected the spontaneous Raman scattering.

\section{EXPERIMENTS}

The experimental setup, depicted in Fig. 1, is nearly the same as those used in previous experiments on multicolor Raman solitons $[15,16]$. As a Kerr waveguide, we use a planar slab waveguide made of a $\mathrm{CS}_{2}$ highly nonlinear liquid layer sandwiched between two SK5 glass blocks and placed in an hermetic tank. The waveguide thickness and length are $12 \mu \mathrm{m}$ and $3 \mathrm{~cm}$, respectively. As a pump laser, we use a compact Q-switched pulsed $\mathrm{Nd}$ :YAG laser with a repetition rate of $1 \mathrm{kHz}$ and pulse width of $450 \mathrm{ps}$. The pump beam is externally frequencydoubled in a KTP crystal which decreases the pulse duration down to $350 \mathrm{ps}$ at $532 \mathrm{~nm}$. It is linearly polarized parallel to the TE mode of the planar waveguide using a Glan polarization beam splitter, which also acts as a variable attenuator with the in-front half-wave plate. We then use an afocal system made of cylindrical lenses for the shaping of an elliptical beam. This elliptical beam is injected in the planar waveguide with a circular lens with a width of $52 \mu \mathrm{m}$ (FWHM) and a height of about $10 \mu \mathrm{m}$. At the waveguide output, we use a CCD camera for direct imaging either the whole output beam or its spectral components by spatially dispersing them using a diffraction grating (2400 lines $/ \mathrm{mm}$ ). We also utilize a streak camera with a resolution of 5 ps for spatiotemporal measurements.

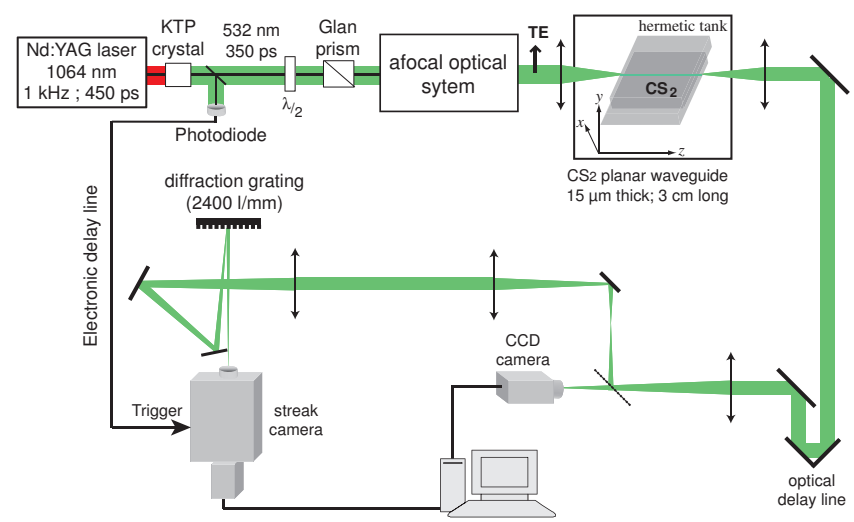

FIG. 1: Experimental setup

\section{A. Spatial analysis: optical Solitons}
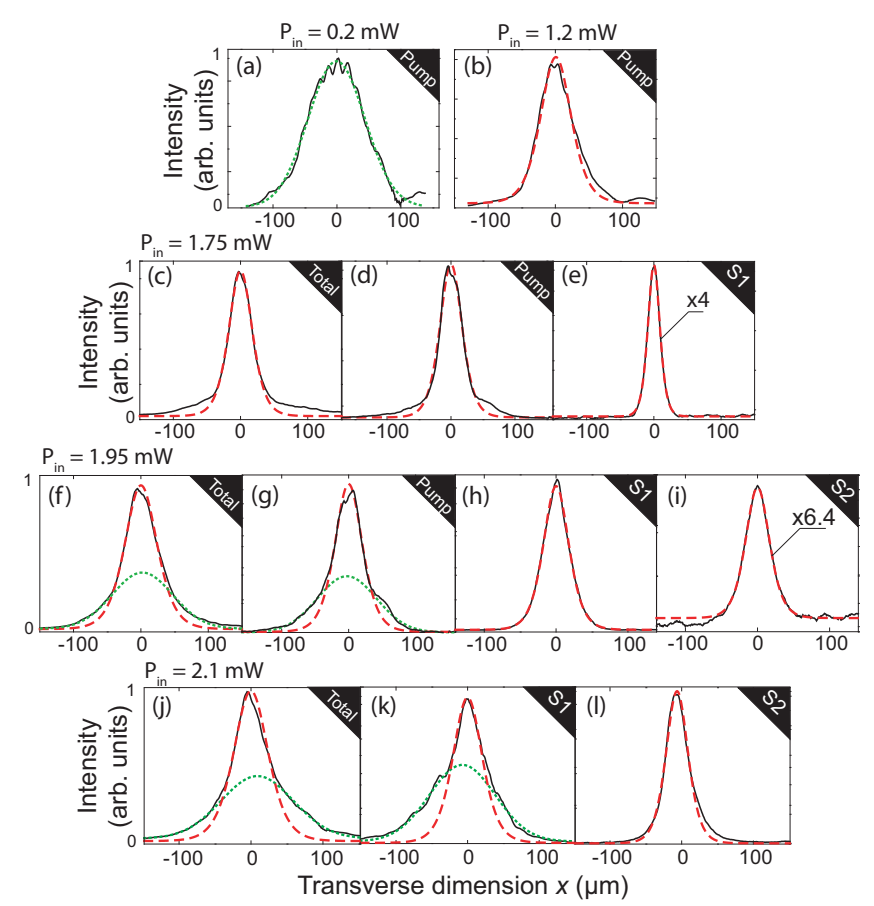

FIG. 2: (color online) Output beams of the waveguide for increasing input pump power from top to bottom. (a) diffraction regime $\left(\mathrm{P}_{i n}=0.2 \mathrm{~mW}\right)$; (b) spatial soliton regime $\left(\mathrm{P}_{i n}=1.2 \mathrm{~mW}\right)$; (c) $\mathrm{P}_{i n}=1.75 \mathrm{~mW}$, with (d) and (e) pump and first-order Raman Stokes S1 beams; (f) $\mathrm{P}_{\text {in }}=1.95 \mathrm{~mW}$, with (g-i) pump, first S1 and second-order S2 Raman beams; (j) $\mathrm{P}_{\text {in }}=2.1 \mathrm{~mW}$, with (k) and (l) S1 and S2 beams. The green dotted and red dashed curves in all subfigures show fits by Gaussian and $\operatorname{sech}^{2}$ functions, respectively.

We first perform a beam analysis by direct imaging the waveguide's output on a CCD camera and by increasing the input pump power. Figures 2 (a) and (b) compare the output beam in the diffraction regime at low pump power and in the spatial soliton regime achieved for an input mean pump power $P_{i n}=1.2 \mathrm{~mW}$, respectively. The output beam in the soliton regime has the same width of $52 \mu \mathrm{m}$ than the input beam and fits very well with the characteristic squared hyperbolic-secant shape $\left(\right.$ sech $\left.^{2}\right)$ of NLS soliton, as shown by the red dashed curve in Fig. 2(b). This 1D optical soliton due to Kerr nonlinearity has already been predicted and observed several decades ago [19-22]. It has been found as a stationary solution of the integrable equation Eq.(1) without the dispersive (third) and Raman (last) terms. Figure 2(c) shows the same beam at a pump power larger than the soliton power. As it can be seen, the beam is narrower than in the soliton regime due to self-focusing. More importantly, after passing the beam through the diffraction grating, we can see in Fig. 2(e) the efficient generation of the first Raman Stokes order labelled S1, which is down-frequency shifted by $20 \mathrm{THz}$ from the pump $\left(\lambda_{S 1}=551 \mathrm{~nm}\right)$. This 
Raman beam also exhibits a $\operatorname{sech}^{2}$ shape with a narrower width than the pump beam. Once generated, the $\mathrm{S} 1$ beam is spatially trapped by the pump beam through XPM and propagates without diffracting. When the Raman beam reaches the same power level than the pump, they are mutually trapped and together form a stable two-color spatial soliton, as that shown by the whole beam plotted in Fig. 2(c). A complete experimental and numerical investigation of this multicolor soliton can be found in Ref. [14]. Here we are interested in how behaves the output beam at higher pump power when cascaded Raman scattering enters into play. Increasing again the input pump power to $P_{i n}=1.95 \mathrm{~mW}$ leads to the generation of a second-order Raman beam at a wavelength of $571 \mathrm{~nm}$ labelled S2 in Fig. 2(i). The S2 beam is still well fitted by a $\operatorname{sech}^{2}$ function with a spatial width close to that of S1, showing that the cascaded Raman process still generates a multicolor spatial soliton. The pump and whole beams however exhibit a strong pedestal fitted by a Gaussian profile (green dotted curve). This pedestal becomes more significant when increasing the pump power till $P_{i n}=2.1 \mathrm{~mW}$, as shown in Figs. $2(\mathrm{j}-\mathrm{k})$. These pedestals are due to the linear and depleted edges of the pump and Raman pulses that spatially diffract and will be thereafter examine through a spatiotemporal analysis.

\section{B. Spatiotemporal analysis: Raman slow light}

We then use in the experimental setup the streak camera to measure the spatiotemporal traces of the spectral components reported in Fig. 2. Figures 3(a-c) show the results of these measurements for the pump, the first S1 and the second S2 Raman components, respectively. The input pump power corresponds to the output beams of Figs. 2(j-l) $\left(P_{\text {in }}=2.1 \mathrm{~mW}\right)$. We can clearly see in Fig. 3(ac) that the three pulses are strongly delayed. The delay between the pump and S1 is about 90 ps whereas that between S1 and S2 is 210 ps. These pulse walk-offs cannot be attributed to GVD that is almost negligible for $3 \mathrm{~m}$ of propagation only. We have indeed calculated that the group delay induced by GVD is only $1.5 \mathrm{ps}$ [15]. Moreover, as GVD is normal, it would generate an advancement instead of an optical delay between the pump and Raman pulses. These optical delays are actually generated by Raman-induced slow light [23]. Because of the narrow linewidth of Raman scattering in $\mathrm{CS}_{2}$, the real part of Raman susceptibility induces a strong group index variation at the Raman frequency, which in turn generates an optical delay. This temporal effect has already been reported for a single Raman soliton (for details, See Ref. [15]). Here we report the observation of cascaded Raman slow light whereby each Raman order generates an optical delay for the next one. Figures 3(d-f) show the results of our numerical simulations for the pump, $\mathrm{S} 1$ and $\mathrm{S} 2$ waves. As it can be seen, the agreement with the experimental measurements of Fig. 3(a-c) is rather
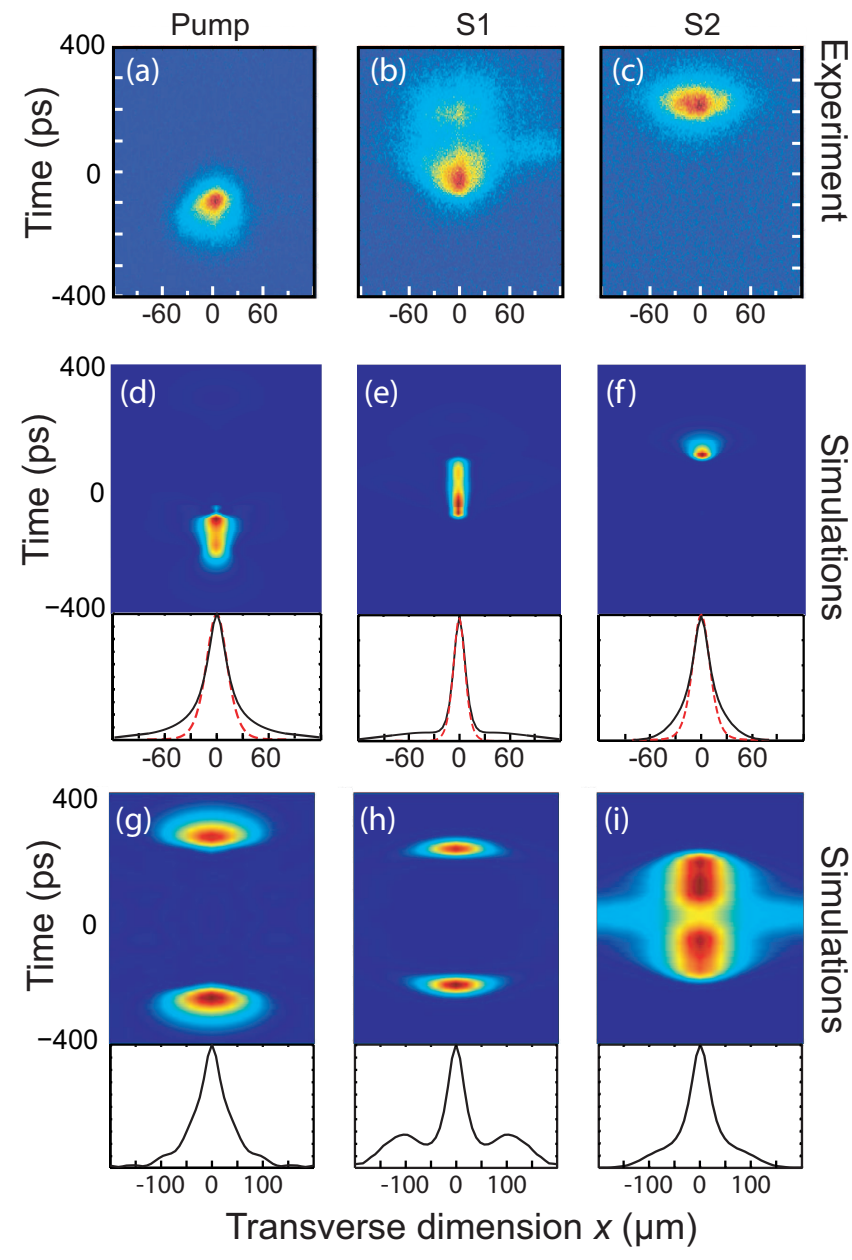

FIG. 3: (color online) Output spatiotemporal profiles for the pump pulse, the first S1 and the second S2 Raman pulses. (a-c): Experimental traces recorded with the streak camera, $P_{i n}=2.1 \mathrm{~mW}$. (d-f): Numerical simulations. (g-i): Numerical simulations without slow light. Bottom insets: normalized time-integrated theoretical spatial profiles (solid black curves) fitted by sech $^{2}$-curve (dotted red curves). $\mathrm{I}=0.7 \mathrm{GW} / \mathrm{cm}^{2}$ for numerical simulations.

good. Theory predicts optical delays between the pump and S1 of $100 \mathrm{ps}$, and 125 ps between S1 and S2, respectively. Moreover, it can be seen that the linear and depleted edges of the pump and Raman pulses diffract and lead to the strong spatial pedestal experimentally observed in Fig. 2 and shown in the bottom of Fig. 3. As a comparison, we also plotted in Figs 3(g-i) the spatiotemporal profiles of the same waves by removing the Raman slow-light process in the numerics. The real part of the Raman susceptibility is simply set to zero. We can see a significantly different spatiotemporal dynamics and, in particular, the pulse splitting due to pump depletion. This comparison highlights the crucial role of slow-light in the cascaded Raman scattering in $\mathrm{CS}_{2}$ and its impact on pulse profiles and beam stability. 
To go further into detail, we numerically investigated both the spatial and temporal dynamics of the pump and Raman pulses by increasing the pump intensity. Figures 4(a-c) and 4(d-f) show the normalized timeintegrated spatial profiles and space-integrated temporal profiles of the pump, S1 and S2 at the waveguide output. The white horizontal solid line corresponds to the spatial soliton intensity $I_{s}$, for which the pump beam generates a spatial soliton and thus conserves its input width all along the waveguide $\left(I_{s}=0.17 \mathrm{GW} / \mathrm{cm}^{2}\right)$. The white horizontal dashed line indicates the intensity threshold from which the pump starts to strongly deplete due to SRS $\left(I_{d}=0.35 \mathrm{GW} / \mathrm{cm}^{2}\right)$. Figure $4(\mathrm{a})$ shows that, below $I_{s}$, the pump beam diffracts and has a wider spatial width than the input beam, whereas self-focusing dominates beyond $I_{s}$. From $I_{d}$ however, the self-focusing stops and the pump beam exhibits a remarkable stability against the pump intensity increase. This is due to the fact that the pump depletes from $I_{d}$ and conserves nearly the same peak intensity to not diffract, as shown in Fig. 4(d) and plotted in Fig. 5 as a black dashed curve. In addition, Fig. 4(e) shows that $\mathrm{S} 1$ is optically delayed by Raman-induced slow-light below $I_{d}$, leading to a time shift towards the trailing edge of the pump. This delay increases with the pump intensity and reaches a maximum of about 90 ps for $I_{d}$. As S1 is delayed with respect to the pump pulse, only the trailing edge of the pump pulse is depleted, as shown in Fig. 4(d). The pump pulse thus conserves a sufficient intensity in its leading edge to still remain a spatially confined beam. This effect is crucial for the generation and stabilization of the spatial multicolor soliton. Beyond $I_{d}$, the spatiotemporal dynamics significantly differs from the undepleted pump regime. Figures 4(e,f) show that both S1 and S2 pulses move towards the leading edge of the pump due to the Raman gain. Therefore, the combined effects of Raman gain and slow-light tend to stabilize the pump beam. On the other hand, S1 continues to grow (See Fig. 5) while decreasing its spatial width (Fig. 4(b)). Depletion of its trailing edge by the $\mathrm{S} 2$ component can be seen in Fig. 4(e) for the input pump intensity of approximately $I_{p}=0.65 \mathrm{GW} / \mathrm{cm}^{2}$. The spatiotemporal dynamics of the $\mathrm{S} 2$ component plotted in Fig. 4(f) is still more complicated. After being amplified and slightly delayed by SRS, S2 is then temporally shifted and localized in the overlapping area between the pump and the first Stokes S1 line (Fig. 4(d-f)) where FWM occurs. As the spectrum of S2 is no more in the Raman gain bandwidth due to the spectral shift generated by the combined effects of walk-off and XPM [24], S2 is actually no more amplified by SRS but rather by FWM involving the pump and S1 [25]. For higher pump intensity than $I_{p}=0.6 \mathrm{GW} / \mathrm{cm}^{2}$, Fig. 4(f) shows that S2 pulse undergoes a fast optical switch due to Raman gain which dominates again FWM gain. S2 is thus localized in the trailing edge of S1. As SRS is more efficient that FWM, a significant increase of the S2 intensity is obtained after $I_{p}=0.6 \mathrm{GW} / \mathrm{cm}^{2}$, as shown in Fig. 5 . This strong Raman conversion leads to the S1 trailing
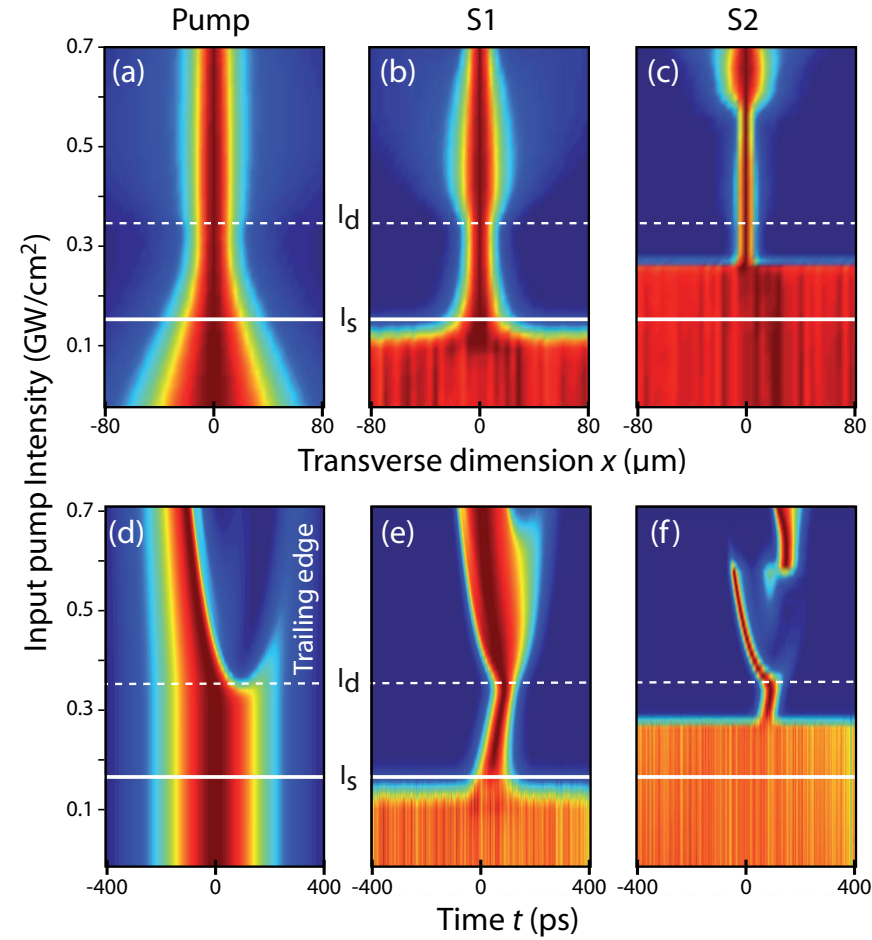

FIG. 4: (color online) Numerical simulations of normalized time-integrated spatial profiles at the waveguide output in function of the input pump intensity for: (a) the pump, (b) the first and (c) the second Stokes order, respectively. (d), (e) and (f) are the corresponding normalized space-integrated temporal profiles. $I_{s}$ : pump intensity for spatial soliton generation for the pump beam only (horizontal white solid line) and $I_{d}$ is the pump depletion intensity threshold (horizontal white dashed line).

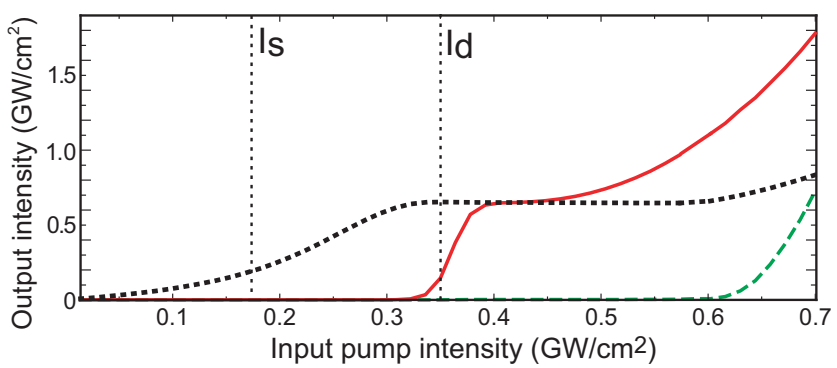

FIG. 5: (color online) Output peak intensity versus the input intensity for the pump (black dots), S1 (red solid curve) and S2 (dashed green).

edge depletion from $I_{p}=0.65 \mathrm{GW} / \mathrm{cm}^{2}$, as shown in Fig. 4(e).

Figures 6(a) and (b) compare the numerical and experimental pulse widths of the three pulses versus the input pump intensity. The gray areas in Figs. 6(a), 7(a) and 8(a) account for numerical values not experimentally reached. After the pump depletion, numerical results of Fig. 6(a) show that the pump pulse duration decreases from $I_{d}$, whereas the $\mathrm{S} 1$ pulse width increases 


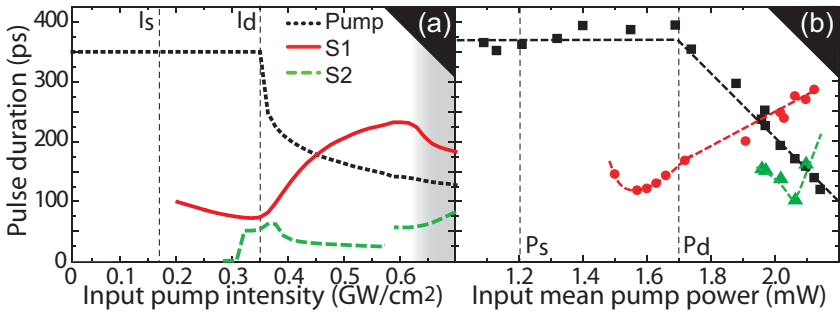

FIG. 6: (color online) (a) Numerical and (b) experimental temporal duration for: the pump in black (dotted curve in (a) and squares in (b)), the S1 component in red (solid curve in (a) and circles in (b)), and the S2 component in green (dashed curve in (a) and triangles in (b)). The dashed curves in (b) serve only to show the trendline.

up to $250 \mathrm{ps}$. The experimental measurements shown in Figs. 6(b) are in relative good agreement with the numerical results. The experimental pump pulse duration however linearly decreases after pump depletion and does not present a stabilization. This behavior could be explained by the fact that S1 is more delayed by slow-light in our experimental data than in the numerical simulations (as we will see thereafter in Fig. 8).

Figure 7 compares the numerical (a) and experimental (b) spatial widths (FWHM) for the three spectral components in function of the input pump intensity. Before the pump depletion intensity $I_{p}$, the pump width decreases due to the self-focusing up to a minimum value around $28 \mu \mathrm{m}$ in numerics and $32 \mu \mathrm{m}$ in experiment. In addition, the S1 width follows the same evolution while remaining below the pump one. This is due to the fact that the S1 component is guided by the pump beam through the Kerr nonlinearity. For the same reason, the S2 beam is much narrower than the pump and S1 components. Near the pump depletion, pump and S1 beams have similar spatial widths. Pump and S1 therefore behave as spatial solitary waves. For higher pump intensity than $I_{d}$, the pump and S1 widths successively increase and decrease whereas numerical simulations predict the stabilization of their spatial widths. No physical process can clearly and

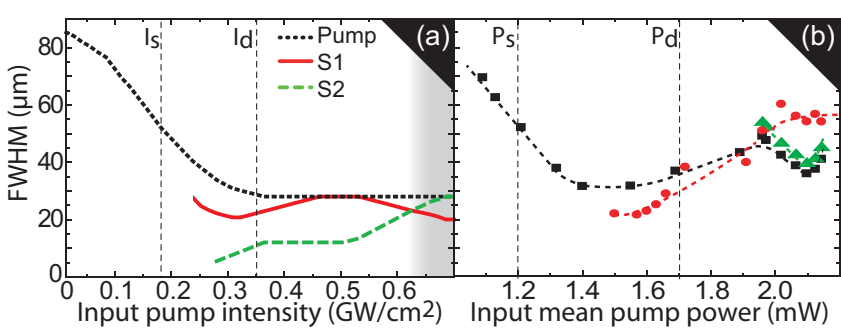

FIG. 7: (color online) (a) Numerical and (b) experimental spatial widths for the pump in black (dotted curve in (a) and squares in (b)), the Raman S1 component in red (solid curve in (a) and circles in (b)), and the Raman S2 component in green (dashed curve in (a) and triangles in (b)). The dashed curves in (b) serve only to show the trendline.

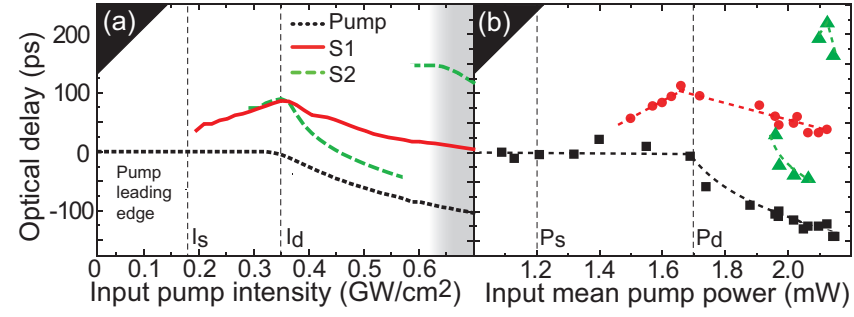

FIG. 8: (color online) (a) Numerical and (b) experimental temporal delay for the pump in black (dotted curve in (a) and squares in (b)), the S1 component in red (solid curve in (a) and circles in (b)), and the S2 component in green (dashed curve in (a) and triangles in (b)). The dashed curves in (b) serve only to show the trendline.

satisfactorily explain this discrepancy between numerical and experimental behavior. This behavior would require further theoretical and experimental studies, which are not the general aim of this paper. Concerning S2, experimental and numerical spatial widths tend to be equal to the pump one for high input pump intensity. This is certainly due to the fact that both components tend to have the same peak intensity as shown in Fig. 5. For the highest pump intensity, the three spectral components exhibit nearly the same spatial width $(30 \mu \mathrm{m}$ for numerical simulations and $50 \mu \mathrm{m}$ for experimental measurements).

Fig. 8 compares the numerical (a) and experimental (b) optical delays measured between the Raman and pump pulses in function of the input pump intensity. As it can be seen, the agreement between both results is quite good. The optical delay of $\mathrm{S} 1$ reaches a maximum value of 90 ps numerically and 110 ps experimentally. Then the S1 delay decreases due to the fact that S1 moves towards the leading edge of the pump. About S2, it is first temporally localized between the pump and the S1 and then undergoes a fast switch, as explained before and clearly observed in our experimental measurements. Figures $9(\mathrm{a}-\mathrm{c})$ and (d-f) show numerical and experimental spatiotemporal profiles of S2 component, respectively, for three different input pump powers. As it can be seen, the agreement between experiment and numerical simulations is excellent. In particular, we measured an optical delay for $\mathrm{S} 2$ of about $250 \mathrm{ps}$ which is close to the numerical value (200 ps). We also clearly observed the splitting and the switch of the $\mathrm{S} 2$ pulse.

From these detailed spatiotemporal analysis, one may wonder if a multicolor spatial soliton is still generated at high pump power level when its components are strongly delayed. As defined in the introduction, a multicolor soliton is formed when all its spectral components are mutually trapped both spatially and temporally. In our case, mutual trapping of the soliton spectral components is stopped by pump depletion and slow light in the strong Raman conversion regime. The nonlinear interaction of the two components is thus reduced to the energy transfer by Raman gain with a weak spatiotemporal overlap. Then, the pump beam is split into two spatial solitons 


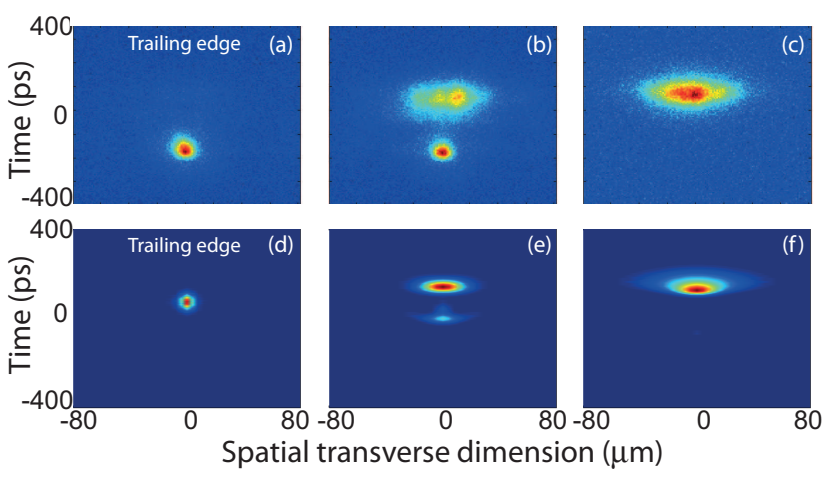

FIG. 9: (color online) Output spatiotemporal profiles of the second Raman Stokes order for three pump powers. Top: experimental measurements for (a) $P_{i n}=2.01 \mathrm{~mW}$, (b) $P_{i n}=$ $2.09 \mathrm{~mW}$ and (c) $P_{\text {in }}=2.12 \mathrm{~mW}$. Bottom: numerical simulations for (d) $I_{i n}=0.57 \mathrm{GW} / \mathrm{cm}^{2}$, (e) $I_{i n}=0.59 \mathrm{GW} / \mathrm{cm}^{2}$ and (f) $I_{i n}=0.61 \mathrm{GW} / \mathrm{cm}^{2}$.

with different frequencies and optically delayed by Raman slow-light. The first Raman order then behaves as a pump and generates in turn its own Raman order to form a new spatial soliton. To resume, the whole beam in the high power regime is no more a strictly speaking multicolor spatial soliton. However several spatial solitons with different colors are successively generated during the propagation. In the time domain, this can be seen as a multicolor pulse train with self-trapped beams.

\section{CONCLUSION}

To conclude, we have investigated both experimentally and numerically the intriguing spatiotemporal dynamics of nonlinear picosecond pulse propagation in a Kerr-type nonlinear planar waveguide. We have demonstrated the formation of multicolor self-trapped optical soliton-like beams through the combined effects of stimulated Raman scattering and cross-phase modulation. It has been shown in particular how Raman-induced slow light plays a crucial role in the beam stability by optically delaying its Raman components. Cascaded Raman slow light was also clearly observed whereby Raman pulses are mutually delayed. Finally, our experimental measurements have been checked against numerical simulations of the spatiotemporal $(1+1 \mathrm{D})$ nonlinear Schrödinger equation. Finally, this work represents the final stage of the characterization of Kerr solitons in nonlinear waveguide in presence of stimulated Raman scattering.

\section{ACKNOWLEDGEMENT}

The authors acknowledge the Conseil Régional de Franche-comté. Computations have been performed on the supercomputer facilities of the Mésocentre de calcul de Franche-Comté.
[1] Z. Chen, M. Segev, D. N. Christodoulides, Rep. Prog. Phys. 75(8):086401 (2012)

[2] G. I. Stegeman and M. Segev, "Optical spatial solitons and their interactions: Universality and diversity," Science 86, 5444, 1518-1523 (1999).

[3] L. Torner and G. I. Stegeman, Multicolor solitons, Optics \& Photonics News 12, 6, 36-39 (June 2001).

[4] Y. S. Kivshar and G. P. Agrawal, Optical solitons (Academic Press, Elsevier Science USA, 2003)

[5] G. Assanto, G. I. Stegeman, Opt. Express 10, 388-396 (2002).

[6] W. E. Torruellas, Z. Wang, D. J. Hagan, E. W. VanStryland, G. I. Stegeman, L. Torner, C. R. Menyuk, Phys. Rev. Lett. 74, 5036-5039 (1995).

[7] L. Torner and G. I. Stegeman, J. Opt. Soc. Am. B 14, p3127 (1997)

[8] Z. Xu, Y. V. Kartashov, L.-C. Crasovan, D. Mihalache, and L. Torner, Phys. Rev. E 70, 066618 (2004)

[9] H. T. Tran, R. A. Sammut, W. Samir, Opt. Commun. 113, 292-304 (1994).

[10] S. Trillo, S. Wabnitz, E. M. Wright, and G. I. Stegeman, Opt. Lett. 13, 871 ?873 (1988).

[11] Y. Chen, J. Atai, Opt. Lett. 19(17), 1287-1289 (1994).

[12] P. B. Lundquist, D. R. Andersen, and Y. S. Kivshar, Phys. Rev. E 57, 3551-3555 (1998).

[13] V. Boucher, and X. Nguyen-Phu, Appl. Opt. 41(21), 4390-4393 (2002).
[14] G. Fanjoux, J. Michaud, M. Delqué, H. Maillotte, T. Sylvestre, Opt. Lett. 31, 3480-3482 (2006).

[15] G. Fanjoux, J. Michaud, H. Maillotte, T. Sylvestre, Phys. Rev. Lett. 100, 013908 (2008).

[16] G. Fanjoux, J. Michaud, M. Delqué, L. Furfaro, H. Maillotte, T. Sylvestre, Opt. Quant. Electron. 40, 271-279 (2008).

[17] G. P. Agrawal, Nonlinear Fiber Optics (Academic, New York, 2001), 3rd ed.

[18] R. W. Boyd, in Nonlinear Optics (Academic Press, Inc., San Diego, 1992), Chapter 9.

[19] R. Y. Chiao, E. Garmire, and C. H. Townes, Phys. Rev. Lett. 13, 479 (1964).

[20] V. E. Zakharov and A. B. Shabat, Zh. Eksp. Teor. Fiz. [Sov. Phys. -JETP 34, 62 (1972)].

[21] A. Barthelemy, S. Maneuf, and C. Froehly, Opt. Commun. 55, 201 (1985).

[22] J. S. Aitchison, A. M. Weiner, Y. Silberberg, M. K. Oliver, J. L. Jackel, D. E. Leaird, E. M. Vogel, and P. W. E. Smith, Opt. Lett. 15, 471-473 (1990).

[23] R. W. Boyd and D.J Gauthier, Progress in Optics 43, 497-530. Elsevier, Amsterdam (2002).

[24] P. L. Baldeck, R. R. Alfano, and G. P. Agrawal, Appl. Phys. Lett. 52, 1939 (1988).

[25] Y. R. Shen and N. Bloembergen, Phys. Rev. 137, A1787 (1965). 